\title{
Tuning the Composition and Porosity of Structured Catalytic Supports for Electrocatalysts Used in Fuel Cells
}

\author{
Byron D. Gates ${ }^{1 *}$, Michael T.Y. Paul ${ }^{1}$, and Brandy Kinkead ${ }^{1}$ \\ 1. Department of Chemistry and 4D LABS, Simon Fraser University, 8888 University Drive, Burnaby, \\ BC, V5A 1S6, Canada \\ * Corresponding author: bgates@sfu.ca
}

Energy produced by solar and wind that is not readily utilized can be stored as mechanical or chemical energy. As an example, the conversion to chemical energy puts an emphasis on the efficiency to which this chemical energy is utilized. Furthermore, the realization of transportation solutions that utilize chemical energy sources that are alternatives to coal or oil require access to efficient means of utilizing stored chemical energy. One area of significant development has been to utilize hydrogen and oxygen molecules as a source of stored chemical energy for both buffering the energy production from alternative sources and to create effective transportation solutions that can compete with internal combustion engines [1]. A growing number of hydrogen fuel cell buses, cars, and other means of transportation are being put in place around the world [2]. Commercially available systems utilize electrocatalysts that are prepared from a combination of carbon nanoparticles supporting platinum based nanocatalysts, along with other essential components such as species to assist in proton transport, or mitigation of oxidative damage or dissolution of the catalyst layers [3]. A critical component to the operation of these electrocatalysts is the ability to efficiently deliver reagents to these reactive sites, and effectively remove the reaction byproducts (i.e., water). The design of commercial electrocatalyst supports is such that they can be produced on large scales, relatively quickly. These methods are effective. There could, however, be further optimization of the catalyst structure and design for utilizing electrocatalytic materials.

The electrocatalyst materials for low temperature fuel cells based on a proton exchange membrane (PEM) technology have utilized a range of platinum based materials, and platinum based nanostructures [4]. These materials include alloys or core-shell type materials of platinum doped with or prepared on other transition metals, such as nickel and cobalt [5]. Aside from a nominally spherical material, these electrocatalysts have taken on a multitude of shapes, such as octahedral, cubic, or hollow particles [6]. A distinct alternative approach is taken through the redesign of the catalyst layer and how the electrocatalysts are utilized therein. The work of $3 \mathrm{M}$ and collaborators produced an ultrathin catalyst layer (UTCL) that contained mesoporous platinum created by physical vapor deposition onto organic templates in a "lostwax" type of templating procedure [7]. Other catalyst layers designs have been pursued that deviate even further from the more widely adopted layers prepared from inks of carbon and platinum nanoparticles. Lessons learned from UTCLs can, however, lead to improved utilization of platinum within catalyst materials and potential a more effective transport of reagents and by-products within these materials.

One alternative approach to the design of the support of the platinum based electrocatalysts is through the creation of structures that have controlled porosities. Porous materials can be created by a lost-wax technique, in which the support material is prepared around a material that serves as a sacrificial template. Removal of that template reveals a scaffold in which the connectivity, and in turn the porosity, depends on the structures and voids of the template material. A template with nanoscale to microscale pores can be prepared by a number of techniques, such as the use of a self-assembled framework that can be 
selectively removed without causing damage to the remaining catalyst support structure [8]. This type of support material has been prepared directly from platinum with utility as a porous catalyst material for the oxygen reduction reaction $[9,10]$. These structures are of interest for their tuned porosity and its correlation to mass transport efficiency and performance of these materials as electrocatalysts.

In this report, we demonstrate the utilization of the platinum as nanocatalysts supported within a porous support, as well as an alternative means of preparing mesoporous platinum UTCLs. The ability to utilize platinum nanocatalysts within alternative catalyst layer designs will provide access to the wealth of established techniques for preparing these nanoparticles. We used spherical, polymer colloids coated with synthetically prepared nanocatalysts, which were self-assembled into an ordered array that served as a template for the formation of the catalyst support. Our approach effectively transfers nanocatalysts to the support matrix during removal of the template material. Porosity and composition of the support materials can be easily tuned from metals to metal oxides to other materials. We also prepared mesoporous UTCLs through the use of additional, self-assembled sacrificial templates. These mesoporous electrocatalyst were similar that prepared by $3 \mathrm{M}$, but utilizing an electrodeposition process that is scalable and tunable. These alternative catalyst layer designs seek an optimal utilization of $\mathrm{Pt}$, and an improved mass transport. Tuning their structure and composition enables a correlation to the efficiency of the oxygen reduction reaction.

We utilize scanning electron microscopy (SEM), scanning transmission electron microscopy (STEM), energy dispersive X-ray spectroscopy (EDS), X-ray fluorescence (XRF) spectroscopy, and inductively coupled plasma techniques for the characterization of the composition and structure of these porous catalyst layers and support materials. Focused ion beam (FIB), SEM, and STEM techniques were also used in our studies to gain additional insights into the structure of these materials both before and after their use in a series of tests to evaluate their utility towards the oxygen reduction reaction and use in catalyst layers of PEM fuel cells or PEMFCs. Additional data is also included for some of these materials to demonstrate their performance in PEMFCs [11].

\section{References:}

[1] C Wang, et al., Energies 9 (2016), p. 603.

[2] B Wu, M Matian and GJ Offer, Int. J. Low-Carbon Technol. 7 (2012), p. 28.

[3] A Kongkan and MF Mathias, Journal of Physical Chemistry Letters 7 (2016), p. 1127.

[4] OT Holton and JW Stevenson, Platin. Met. Rev. 57 (2013), p. 259.

[5] X Zhang, S Yu, L Qiao, W Zheng and P Liu, J. Chem. Phys. 142 (2015), p. 194710.

[6] L Dubau, et al. J. Mater. Chem. A 2 (2014), p. 18497.

[7] DF van der Vliet, et al., Nat. Mater. 11 (2012), p. 1051.

[8] B Gates, Y Yin and Y Xia, Chem. Mater. 11 (1999), p. 2827.

[9] B Kinkead, et al., Electrocatalysis 4 (2013), p. 179.

[10] OH Kim, et al., Nat. Commun. 4 (2013), p. 2473.

[11] This research was financially supported in part by from the Natural Sciences and Engineering Research Council (NSERC) Discovery Program (Grant No. 1077758), CMC Microsystems (MNT Grant No. 4440), and the Canada Research Chairs Program (B.D. Gates; Grant No. 950-215846). This work made use of 4D LABS (www.4dlabs.ca) and the Centre for Soft Materials shared facilities supported by the Canada Foundation for Innovation (CFI), British Columbia Knowledge Development Fund (BCKDF), Western Economic Diversification Canada, and Simon Fraser University. 